Check for updates

Cite this: RSC Adv., 2019, 9, 14194

Received 31st January 2019

Accepted 1st May 2019

DOI: $10.1039 / \mathrm{c} 9 \mathrm{ra00847k}$

rsc.li/rsc-advances

\section{Synthesis of discrete catalytic oligomers and their potential in silica-supported cooperative catalysis $\uparrow$}

\author{
Prakash Chandra, ${ }^{a}$ Alain M. Jonas (D) a and Antony E. Fernandes (D) *ab \\ Cooperative catalysis on solid surfaces relies primarily on two or more catalytic partners being close enough \\ to each other to sustain the catalytic cycle. We describe here the synthesis and preliminary investigation of \\ discrete homo-oligomers as flexible scaffolds to inflect the intersite distance and blur the compositional \\ heterogeneity in a silica-grafted catalytic triad.
}

Supported multifunctional catalysts provide significant advantages over their parent homogeneous systems for more sustainable and efficient approaches in cooperative and cascade catalysis. ${ }^{1-12}$ However, although it is now becoming possible to combine most activation modes on a single support and compete with the original molecular catalysts, controlling the surface distribution of the different functional groups remains challenging. ${ }^{13-18}$ As exemplified by biological catalysts, the perfect spatial preorganization of catalytic centers within a controlled and confined environment is central to enable optimal synergistic interactions. Hence, the current limitation of grafting strategies to random, entropy-controlled systems is an important issue that prevents the fully rational design of active multifunctional surfaces.

The utilization of mixed monolayers for the preparation of silica-supported multifunctional catalysts intrinsically leads to a statistical distribution of the active components on surface. ${ }^{19}$ This eventually limits the potential of these systems since it creates a statistical distribution of pairings and spacings between the functional groups, with some catalytic partners being found at unmatched stoichiometry and distance within the mixed monolayer. Hence, it becomes important to develop methods to optimize the spatial positioning of functional groups in supported multifunctional catalysts to overcome the limitations associated with random functionalization. So far, only a few strategies have been proposed to reach a more homogeneous spatial distribution of multiple functional groups on surface. Molecular imprinting or imprint coating methods have allowed to better control the relative arrangement of surface-bound functional groups. ${ }^{20-25}$ For instance, Davis demonstrated that paired thiol/sulfonic acid obtained by molecular imprinting on silica surpassed the same randomly

${ }^{a}$ Institute of Condensed Matter and Nanosciences, Université catholique de Louvain, 1348 Louvain-la-Neuve, Belgium

${ }^{b}$ Certech, Rue Jules Bordet 7180 Seneffe, Belgium. E-mail: antony.fernandes@certech. be

$\dagger$ Electronic supplementary information (ESI) available. See DOI: 10.1039/c9ra00847k distributed cooperative catalytic system. ${ }^{23}$ In another way, the complementary functional groups can be assembled on a single molecular scaffold prior to immobilization. ${ }^{26}$ In this direction, we recently reported the use of short sequence-defined oligomers for the preorganization of the (pyta)Cu/TEMPO/imidazole (pyta $=$ pyridyltriazole, TEMPO $=2,2,6,6$-tetramethylpiperidine$N$-oxyl) catalytic triad on mesoporous silica and their superior activity in the aerobic oxidation of alcohols compared to a mixed monolayer of the triad. ${ }^{27,28}$

The homogeneous (bpy)Cu/TEMPO/NMI catalyst system (bpy = bipyridine, NMI $=N$-methyl-imidazole) developed by Stahl and coworkers, ${ }^{29-32}$ provides a practical, efficient and environmentally benign alternative compared to common methods based on stoichiometric and toxic oxidizing reagents for the transformation of alcohols to aldehydes and ketones. ${ }^{33}$ The mechanism involves the formation of a (bpy) $\mathrm{Cu}^{\mathrm{I}}(\mathrm{NMI})$ complex that is then oxidized by molecular oxygen to provide the active oxidizing $\mathrm{Cu}^{\mathrm{II}}$ species. The role of TEMPO is still unclear as whether it interacts directly or not with the $\mathrm{Cu}$ center; ${ }^{34-36}$ however, the formation of the (bpy) $\mathrm{Cu}^{\mathrm{I}}(\mathrm{NMI})$ complex remains central in the catalytic cycle. Hence, in our approach, it is important to promote interactions between the supported $\mathrm{Cu}$ and imidazole sites while the TEMPO is located in close vicinity of the $\mathrm{Cu}$ complex to enable high turnovers. Here, we present another approach to minimize the effect of local compositional heterogeneity in randomly-mixed monolayers, based on the use of multivalent flexible short chains which dynamically blur the local composition of the grafted layer, and thereby achieve a statistically more efficient pairing between functional units.

In that aim, we first describe the simple iterative synthesis of azide-functionalized monodisperse oligomers as versatile platforms for the anchoring of the three catalytic units (pyta) $\mathrm{Cu}$, TEMPO and imidazole. After grafting a stoichiometric mixture of the oligomers on mesoporous silica, we find that the catalytic activity per unit amount of catalytic center is strongly enhanced for longer oligomeric chains. This is ascribed to the larger volume explored by longer chains, resulting in a more efficient coupling between the three elements of the catalytic triad despite the grafting heterogeneity of the layers. The functional homo-dimers 13, 15 and 17 and homo-trimers 14, 16 and 18 
bearing pendant TEMPO, imidazole and pyta active centers, respectively, share the identical dimeric and trimeric precursors 9 and 10, respectively (Scheme 1). The oligomeric platforms 9 and 10 were straightforwardly synthesized from racemic propargyl glycidyl ether $\mathbf{1}$ following an iterative epoxide opening/ CuAAC (copper-catalyzed azide-alkyne cycloaddition) sequence. ${ }^{27,37,38}$ Precisely, the opening of the oxirane ring in $\mathbf{1}(n$ $=0$ ) with the azide anion releases a secondary hydroxyl function that would later serve for the installation of the catalytic units. The azide functionality in 2 subsequently allows the reintroduction of the epoxide motif by CuAAC reaction with propargyl glycidyl ether. Reiterating this elongation sequence allows to access monodisperse oligomers with lateral $-\mathrm{OH}$ handles.

The "living" oligomers $\mathbf{4}$ and $\mathbf{6}$ were terminated by reaction of the end-chain azide with phenylacetylene under CuAAC conditions to afford dimer 7 and trimer 8, respectively. In order to install the catalytic components of the triad, the lateral hydroxyl groups were treated with $N, N^{\prime}$-disuccinimidyl carbonate (DSC) followed by coupling with 2-azidoethylamine, to provide the dimeric (9) and trimeric (10) platforms with accessible azide groups. The later were reacted either with TEMPO 11, imidazole 12, or 2-ethynylpyridine under CuAAC conditions to provide the TBDMS-protected multivalent homooligomers that were ultimately treated with TBAF (tetra- $n$ - butylammonium fluoride) to afford the alkyne-terminated oligomers 13-18.

The two-step post-polymerization modification strategy developed here for the installation of lateral functional groups mirrors previous approaches based on labile ester bounds. ${ }^{27,37}$ Precisely, the lateral carbamate bounds ensure here enhanced chemical stability compared to ester analogues that are partly or totally cleaved during the TBAF-mediated alkyne deprotection while the carbamate variants remain unaltered.

Trifunctional (pyta)Cu/TEMPO/imidazole supported catalysts of increasing valency were prepared using our typical CuAAC protocol by reacting stoichiometric mixtures of the corresponding alkyne-terminated catalytic groups with azidefunctionalized mesoporous silica in presence of $\mathrm{Cu}(\mathrm{I})$ (Scheme 2); the $\mathrm{Cu}(\mathrm{I})$ used for the CuAAC grafting also serving for the realization of the (pyta)Cu sites of the supported catalysts. ${ }^{39,40}$

In order to further study the effect of the oligomeric scaffolds, the more traditional "monomeric" supported catalyst 19 was prepared from 11, 12 and 2-ethynylpyridine (1: 1 : 1 molar ratio) and azide silica $(0.29 \pm 0.04 \mathrm{mmol}$ azide per $\mathrm{g})$ in presence of $\mathrm{CuI}$ and $\mathrm{Et}_{3} \mathrm{~N}$.

Similarly, catalyst 20 was obtained by reacting a mixture of dimers 13, 15 and 17 in a $1: 1: 1$ molar ratio with azide silica; catalyst $\mathbf{2 1}$ was in turn prepared from a stoichiometric mixture of trimers 14, 16 and 18.

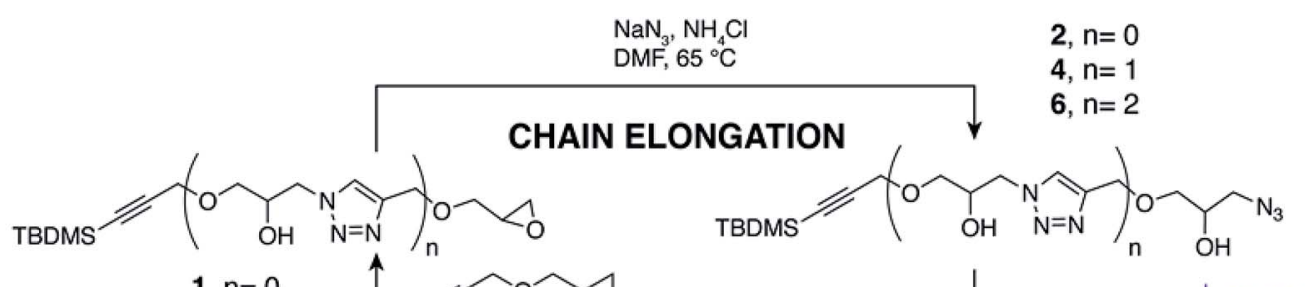

$1, \mathrm{n}=0$

$3, n=1$



$5, n=2$ DMF, $55^{\circ} \mathrm{C}$

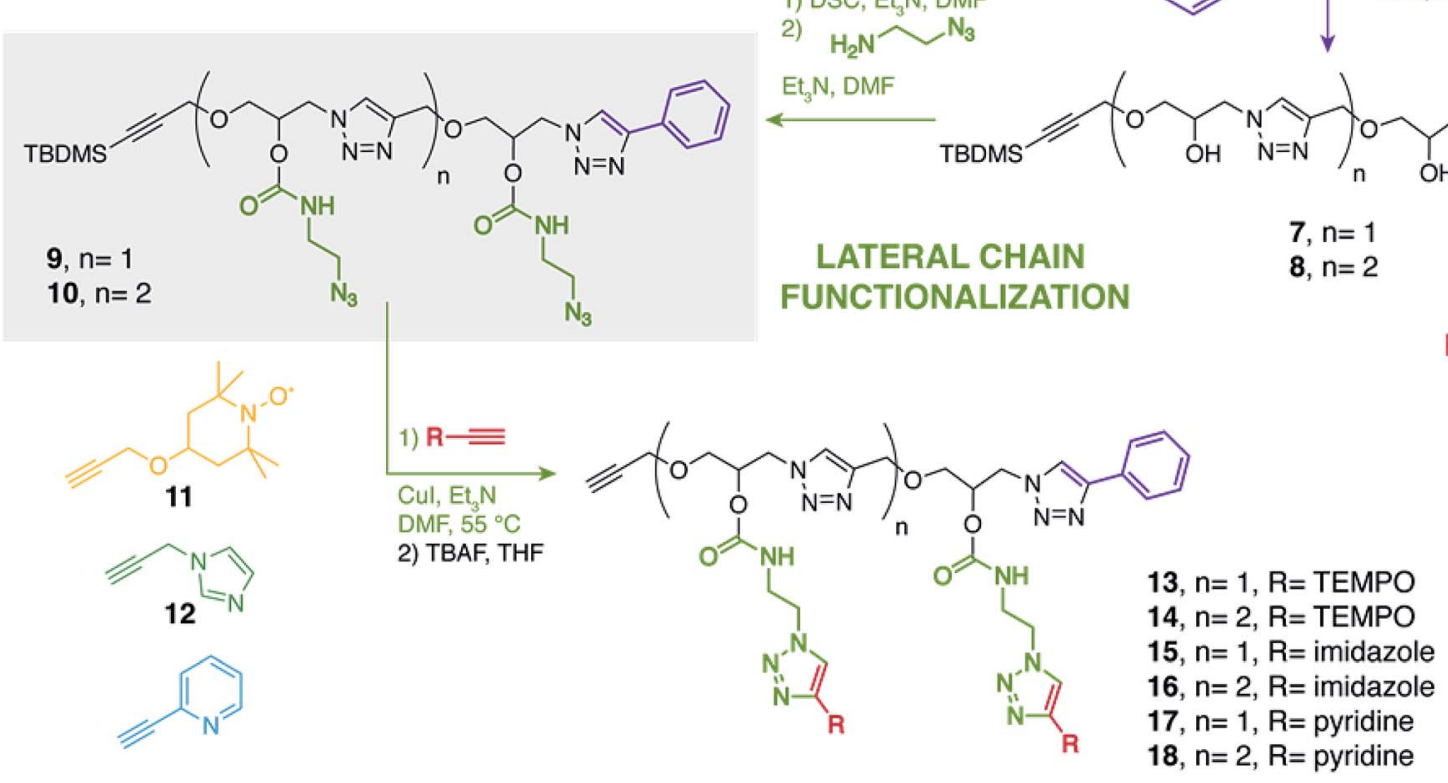

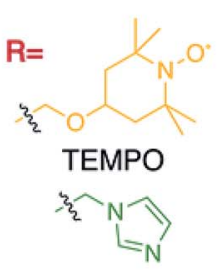

imidazole<smiles>[Y]c1ccccn1</smiles>

pyridine

Scheme 1 Preparation of the discrete catalytic oligomers 13-18 


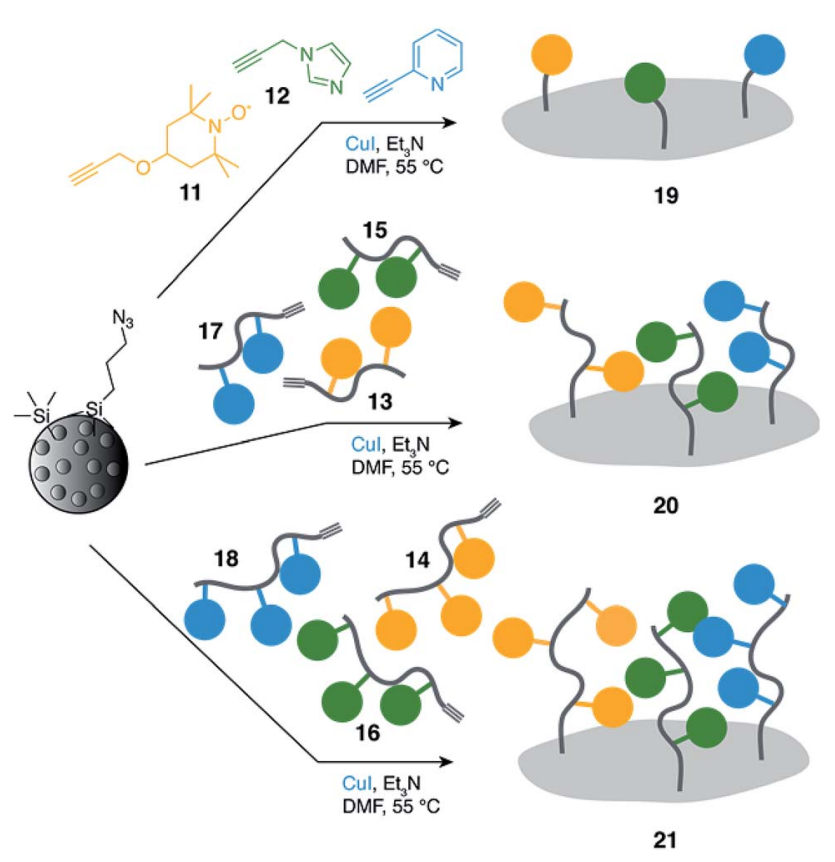

Scheme 2 Preparation of silica-supported multifunctional catalysts 19-21.

The grafting reactions were monitored by thermogravimetric analysis (TGA) and FT-IR. TGA (ESI, Fig. S41a †) provided loadings of $0.27 \pm 0.01,0.15 \pm 0.01$ and $0.12 \pm 0.01 \mathrm{mmol} \mathrm{g}^{-1}$ for 19, 20 and 21, respectively, which corresponds to grafting efficiencies of $89 \pm 11,53 \pm 11$ and $43 \pm 9 \%$ with respect to the initial azide loading. The DTG curves (ESI, Fig. S41b $\dagger$ ) also showed the persistence of thermal events corresponding to the azide layer for catalysts $\mathbf{2 0}$ and 21, also indicative of partial grafting. As can be expected, the grafting efficiency decreased with the length and bulkiness of the oligomers as steric hindrances enter into consideration for the surface CuAAC reaction. FT-IR confirmed this observation with the residual azide signal $\left(2100 \mathrm{~cm}^{-1}\right)$ being more prominent with the length of the immobilized oligomers, synonymous with incomplete CuAAC grafting (ESI, Fig. $\mathrm{S} 42 \dagger)$. The apparition of a $\mathrm{C}=\mathrm{O}$ vibration band at $1730 \mathrm{~cm}^{-1}$ in the FT-IR spectra of 20 and 21 provided additional evidence for the covalent grafting of the multifunctional oligomers bearing carbamate bounds (Fig. S42†). The difference between the loadings of di- and trimeric chains is small, which results from their (unperturbed) radii of gyration being close (the theoretical ratio being $(2 / 3)^{1 / 2}=0.82$ ). In contrast, the "monomeric" units are essentially rigid rods that can be grafted to much higher densities. The content of $\mathrm{Cu}$ for each silica catalysts was quantified by ICP-AES, giving Cu loadings of 0.08 , 0.09 and $0.10 \mathrm{mmol} \mathrm{g}^{-1}$ for 19,20 and 21 , respectively. The experimental $\mathrm{Cu}$ contents fit well with the theoretical values calculated from the measured grafting density and the expected formation of a $1: 1$ pyta/Cu complex (0.09, 0.10 and $0.12 \mathrm{mmol}$ $\mathrm{g}^{-1}$ for 19, 20 and 21, respectively). Noticeably, owing to the reduction of grafting efficiencies with chain length, catalysts 19-21 have nearly identical $\mathrm{Cu}$ loadings. XPS analysis (ESI, Fig. S43†) of supported catalysts 19-21 confirmed the presence of $\mathrm{Cu}(\mathrm{I})$ species with $\mathrm{Cu} 2 \mathrm{p}_{1 / 2}$ and $\mathrm{Cu} 2 \mathrm{p}_{3 / 2}$ peaks at $c a .952 .4$ and
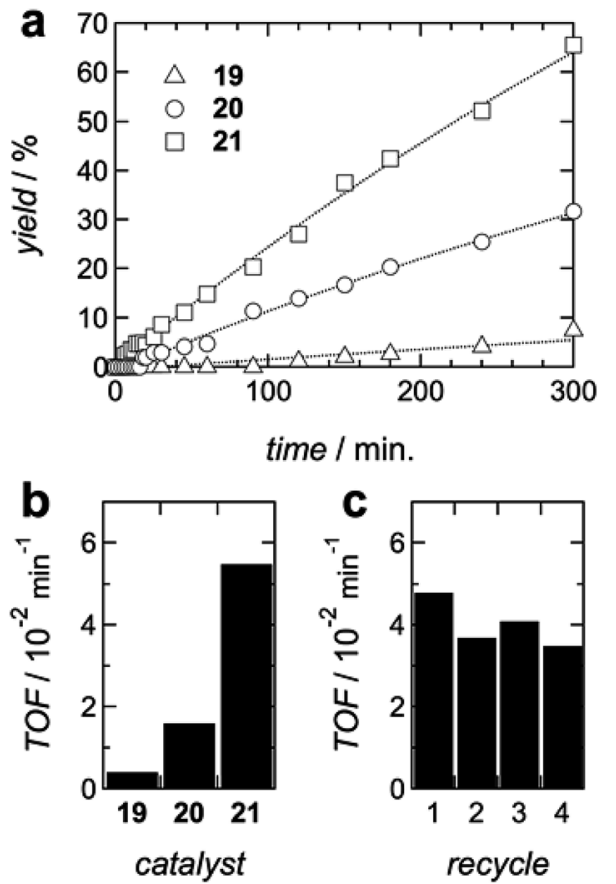

Fig. 1 (a) Activity of catalysts 19-21 (5 mol\% based on $\mathrm{Cu}$ ) in the aerobic oxidation of benzyl alcohol. Conditions: $\mathrm{BnOH}(0.2 \mathrm{mmol})$ in acetonitrile $(0.2 \mathrm{M}), \mathrm{O}_{2}$ bubbling $\left(5.5 \mathrm{~mL} \mathrm{~min}^{-1}\right), 60^{\circ} \mathrm{C}$. (b) Initial turnover frequencies (TOFs). (c) Recycle of catalyst 21.

$932.5 \mathrm{eV}$, respectively (Fig. S44 $\dagger$ ). In addition, analysis of the high-resolution XPS C 1s region showed an increase of the highenergy components at $c a$. $286.4 \mathrm{eV}$ and the apparition of a peak of higher energy at $289.0 \mathrm{eV}$ in catalysts 20 and 21 compared to 19, which are attributed to the extra electron deficient $\mathrm{C}-\mathrm{O}$ and $\mathrm{C}=\mathrm{O}$ carbons of the oligomers, respectively (ESI, Fig. S44 $\dagger$ ).

The supported catalysts were tested in the model aerobic oxidation of benzyl alcohol ${ }^{29,30}$ using a 5 mol\% $\mathrm{Cu}$ loading (Fig. 1a). Catalyst 21, with the mixed homo-trimer monolayer, showed the highest activity with an initial turnover frequency (TOF) more than one order of magnitude superior to the more traditional mixed monolayer catalyst 19 (Fig. 1b).

Catalyst 20 showed itself a 4-fold increase in initial TOF compared to 19 . Catalyst 21 could be easily recovered and reused for five consecutive runs with moderate erosion of its activity (Fig. 1c).

The remarkable increase of catalytic efficiency of the oligomeric chains can only be explained by the local mixing of the three catalytic units, which is provided by the flexibility of the chains combined with the larger volume of space statistically sampled by the random fluctuations of longer chains. In contrast, the rigid monomeric units do not permit an optimal pairing of the catalytic centers, resulting in a strongly depreciated TOF.

\section{Conclusions}

In conclusion, we described here the synthesis of short discrete oligomers using an iterative epoxide opening/CuAAC chemistry. Derivatization of the resulting lateral hydroxyl groups into azide 
handles provided unique platforms for the integration of catalytic units. Homo-dimers and -trimers bearing the (pyta) $\mathrm{Cu}$, TEMPO and imidazole active centers were obtained and grafted on mesoporous silica. The supported oligomeric catalysts surpassed the activity of a more conventional mixed monomeric version of the triad, with the trimeric catalysts showing a 14 -fold enhanced TOF in the model aerobic oxidation of benzyl alcohol.

The utilization of oligomeric scaffolds opens opportunities in supported cooperative catalysis by affording ways to expand the probability of synergistic interactions within confined spaces. Further refining the tacticity of the chains and the relative arrangement of the pendant functional groups is under investigation.

\section{Conflicts of interest}

There are no conflicts to declare.

\section{Acknowledgements}

The authors acknowledge the European Regional Development Fund (ERDF) and Wallonia (Operational Program "Wallonia2020.EU”), the Belgian Federal Science Policy (IAP P7/05) and the Fonds de la Recherche Scientifique - FNRS and the Fonds Wetenschappelijk Onderzoek under EOS project no. 30650939 for financial support. Anne Iserentant, Cécile D'Haese and François Devred are acknowledged for ICP-AES, XPS and physisorption measurements, respectively. Gabriella BarrozinoConsiglio is acknowledged for help with some NMR measurements.

\section{Notes and references}

1 J. M. Notestein and A. Katz, Chem.-Eur. J., 2006, 12, 39543965.

2 E. L. Margelefsky, R. K. Zeidan and M. E. Davis, Chem. Soc. Rev., 2008, 37, 1118-1126.

3 F.-X. Felpin and E. Fouquet, ChemSusChem, 2008, 1, 718-724.

4 J.-K. Lee, M. Kung and H. Kung, Top. Catal., 2008, 49, 136144.

5 S. Shylesh and W. R. Thiel, ChemCatChem, 2011, 3, 278-287.

6 C. Yu and J. He, Chem. Commun., 2012, 48, 4933-4940.

7 U. Diaz, D. Brunel and A. Corma, Chem. Soc. Rev., 2013, 42, 4083-4097.

8 N. A. Brunelli and C. W. Jones, J. Catal., 2013, 308, 60-72.

9 K. Motokura, ChemCatChem, 2014, 6, 3067-3068.

10 M. J. Climent, A. Corma, S. Iborra and M. J. Sabater, ACS Catal., 2014, 4, 870-891.

11 D. Jagadeesan, Appl. Catal., A, 2016, 511, 59-77.

12 Y.-B. Huang, J. Liang, X.-S. Wang and R. Cao, Chem. Soc. Rev., 2017, 46, 126-157.

13 A. Puglisi, R. Annunziata, M. Benaglia, F. Cozzi, A. Gervasini, V. Bertacche and M. C. Sala, Adv. Synth. Catal., 2009, 351, 219-229.

14 C.-H. Tsai, H.-T. Chen, S. M. Althaus, K. Mao, T. Kobayashi, M. Pruski and V. S. Y. Lin, ACS Catal., 2011, 1, 729-732.
15 N. A. Brunelli, K. Venkatasubbaiah and C. W. Jones, Chem. Mater., 2012, 24, 2433-2442.

16 N. A. Brunelli, S. A. Didas, K. Venkatasubbaiah and C. W. Jones, J. Am. Chem. Soc., 2012, 134, 13950-13953.

17 S. Shylesh, D. Hanna, J. Gomes, S. Krishna, C. G. Canlas, M. Head-Gordon and A. T. Bell, ChemCatChem, 2014, 6, 1283-1290.

18 H. Noda, K. Motokura, Y. Wakabayashi, K. Sasaki, H. Tajiri, A. Miyaji, S. Yamaguchi and T. Baba, Chem.-Eur. J., 2016, 22, 5113-5117.

19 R. Mouawia, A. Mehdi, C. Reye and R. J. P. Corriu, J. Mater. Chem., 2008, 18, 4193-4203.

20 A. Katz and M. E. Davis, Nature, 2000, 403, 286.

21 V. Dufaud and M. E. Davis, J. Am. Chem. Soc., 2003, 125, 9403-9413.

22 J. D. Bass and A. Katz, Chem. Mater., 2006, 18, 1611-1620.

23 E. L. Margelefsky, A. Bendjériou, R. K. Zeidan, V. Dufaud and M. E. Davis, J. Am. Chem. Soc., 2008, 130, 13442-13449.

24 Y. Peng, J. Wang, J. Long and G. Liu, Catal. Commun., 2011, 15, 10-14.

25 J. E. Lofgreen and G. A. Ozin, Chem. Soc. Rev., 2014, 43, 911933.

26 X. Yu, X. Yu, S. Wu, B. Liu, H. Liu, J. Guan and Q. Kan, J. Solid State Chem., 2011, 184, 289-295.

27 P. Chandra, A. M. Jonas and A. E. Fernandes, J. Am. Chem. Soc., 2018, 140, 5179-5184.

28 P. Chandra, A. M. Jonas and A. E. Fernandes, ACS Catal., 2018, 8, 6006-6011.

29 J. M. Hoover and S. S. Stahl, J. Am. Chem. Soc., 2011, 133, 16901-16910.

30 J. M. Hoover, J. E. Steves and S. S. Stahl, Nat. Protoc., 2012, 7, 1161-1166.

31 J. M. Hoover, B. L. Ryland and S. S. Stahl, J. Am. Chem. Soc., 2013, 135, 2357-2367.

32 J. M. Hoover, B. L. Ryland and S. S. Stahl, ACS Catal., 2013, 3, 2599-2605.

33 J.-E. Bäckvall, Modern Oxidation Methods, Wiley-VCH Verlag \& Co. KGaA, 2nd edn, 2010.

34 B. L. Ryland, S. D. McCann, T. C. Brunold and S. S. Stahl, J. Am. Chem. Soc., 2014, 136, 12166-12173.

35 R. C. Walroth, K. C. Miles, J. T. Lukens, S. N. MacMillan, S. S. Stahl and K. M. Lancaster, J. Am. Chem. Soc., 2017, 139, 13507-13517.

36 J. Rabeah, U. Bentrup, R. Stosser and A. Bruckner, Angew. Chem., Int. Ed. Engl., 2015, 54, 11791-11794.

37 J. C. Barnes, D. J. C. Ehrlich, A. X. Gao, F. A. Leibfarth, Y. Jiang, E. Zhou, T. F. Jamison and J. A. Johnson, Nat. Chem., 2015, 7, 810-815.

38 Y. Jiang, M. R. Golder, H. V. T. Nguyen, Y. Wang, M. Zhong, J. C. Barnes, D. J. C. Ehrlich and J. A. Johnson, J. Am. Chem. Soc., 2016, 138, 9369-9372.

39 A. E. Fernandes, O. Riant, A. M. Jonas and K. F. Jensen, RSC Adv., 2016, 6, 36602-36605.

40 A. E. Fernandes, O. Riant, K. F. Jensen and A. M. Jonas, Angew. Chem., Int. Ed., 2016, 55, 11044-11048. 